\title{
Ab-initio study of electrical and optical properties of allylamine
}

\author{
B. Dec ${ }^{1}$, K. Pyrchla ${ }^{1}$, R. Bogdanowicz ${ }^{1}$ \\ ${ }^{1}$ Faculty of Electronics, Telecommunications and Informatics, Gdańsk University of Technology, G. \\ Narutowicza 11/12, 80-233 Gdańsk
}

Received September 10, 2018; accepted September 12, 2018; published September 30, 2018

\begin{abstract}
The Density functional theory is one of the most promising methodologies in fast and accurate calculations of electrical and optical properties from the atomic basis. In this paper, we calculate the electrical and optical properties of allylamine (2-propen 1- amine) in terms of accuracy and speed of calculations obtained by selection of the DFT-1/2 method with ultrasoft Vanderbilt pseudopotentials. The comparison of density of states between the molecule and the bulk configuration shows great agreement between them, therefore we calculated the refractive index which showed even better agreement with experimental data.
\end{abstract}

Density functional theory (DFT) [1] is an attractive tool for predicting materials properties and comparing that data with experimental results. It achieves satisfactory agreement to the various experiments, which brings this method as one of most interesting in the scientific community. DFT is also is cost effective and enables to calculate larger and more complex structures over the last period. It is popular for being quantum scale simulation method revealing the electronic properties of semiconducting materials. In principle, DFT describes the Pauli phenomena in the multi-electron system by approximating exchange-correlation potentials. Overall, the most actual approach engages General Gradient Approximation (GGA) with density functional described by Perdew, Burke, and Ernzerhof (PBE) [2]. The Linear Combination of Atomic Orbitals (LCAO) [3] technique with exchange and correlation within Kohn-Sham DFT formalism was here particularly used. The GGA with PBE density functional were jointly utilized to acquire relaxed structure of allylamine. For electrical and optical properties we use GGA-1/2 with PBE method in order to obtain self-correction of DFT self-interaction error.

This method defines atomic self-energy potential in a way that cancels electron-hole self-interaction energy. It has been found to greatly improve bandgaps for a wide range of semiconductors and isolators [4]. We have also used Optimized Norm-Conserving Vanderbilt (SG15) pseudopotentials [5].

The absorption of allylamine causes only minimal changes in the bonds and geometrical structures of atomic clusters, therefore provide sites for the covalent immobilization of graft polymers and biomolecules on chemically inert surfaces [6-7]. One of the most important properties is that the number of absorbed allylamine molecules to the surface can affect the optical spectrum [8]. Therefore, it gives an enhancement of sensitivity in long-period fiber gratings [9]. The characteristic of surface terminated with an adsorbed allylamine molecule can be adjusted and therefore results in controlling the optical properties of the material. In general, amine enhances absorbance and fluorescence. Another interesting property is hydrophilic and therefore can be used to produce useful for solution phase sequential reactions with various aldehydes and carboxylic acids. These properties make allylamine an important material in fabricating hybrid devices and sensors.

Our experiments with the allylamine structure are a base point for future calculations of the electro-optical properties of a thin film of organic material on a BDD structure. The results of the dispersion calculations of pristine allylamine indicate that the presented method may be used in the design process of materials for electrooptical identification [10].

An allylamine structure was created using a 3D molecule builder in software Atomistic Toolkit 2018.6 [11] from Synopsys.

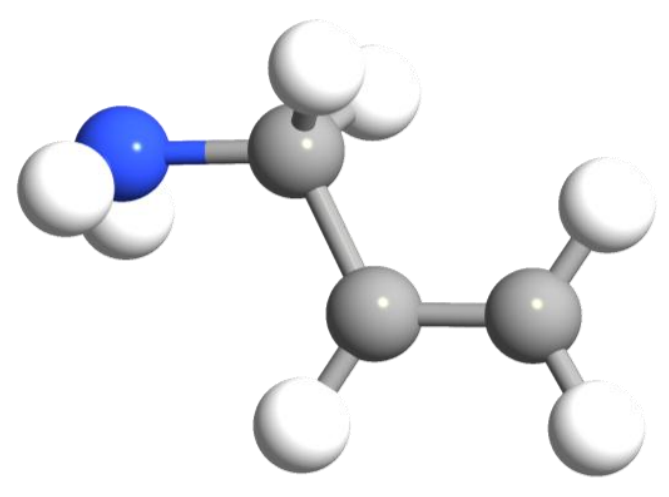

Fig. 1. 3D molecule visualization of an allylamine molecule after optimization of forces with an L-BFGS algorithm. Between two right carbon atoms we can observe a shorter bond which is the representation of a double bond. 
Relaxation of an allylamine molecule and surface slab were done with a limited memory Broyden-FletcherGoldfarb-Shanno (BFGS) algorithm [12].

The structure has been optimized with a forces tolerance level below $0.01 \mathrm{eV} / \AA$. Figure 1 illustrates the allylamine molecule in a $3 \mathrm{D}$ ball and stick representation.

The mesh cutoff energy was set to 370 Rydberg and the kpoint sampling was Monkhorst-Pack grid with $x, y, z$ (16, 20,30). The boundary conditions were periodic in all directions and the Poisson solver was a fast Fourier solver. The tolerances in total band energy and the hamiltonian matrix elements were set to $10^{-5} \mathrm{eV}$. The vacuum buffer of $10 \AA$ surrounding the allylamine molecule model was used to prevent interaction between molecules due to periodic boundary conditions. The structure with periodic boundary conditions was used in computation because the properties such as refractive index, extinction coefficient, absorption cannot be calculated using molecule configuration in Atomistic Toolkit 2018.6 [11] from Synopsys. For calculations, we use SG15 pseudopotentials [5].

The Density of States (DOS) calculation for the allylamine is presented in Fig. 2, where we can observe the comparison of two DOS plots computed for the molecular configuration and the bulk configuration of allylamine.

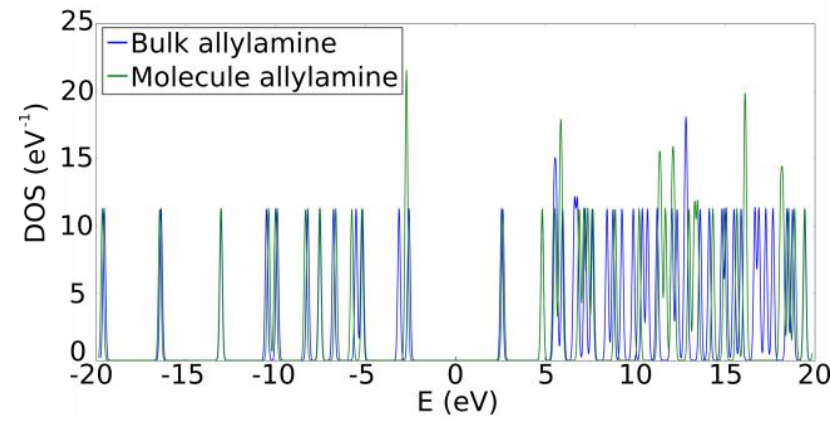

Fig. 2. The density of states for allylamine in a) molecule configuration (green) and b) bulk configuration with 10A spacing (blue).

The key point during this selection was to obtain maximum accuracy with chosen methods, which is possible if we take into account more atomic shells [13] but do not exceed the moment when the computational cost increases rapidly without any significant change in accuracy. Figure 3 presents selected pseudopotentials for each calculated basic element.

The first calculation performed for a pure allylamine model was the density of states calculation. It is widely known that optical properties of matter depend on its electron structure. If we want to receive well-converged data about the optical properties of a material, we must know that we have the right model of its electron structure. The precision of the calculation of electron properties indicates the precision of optical properties.
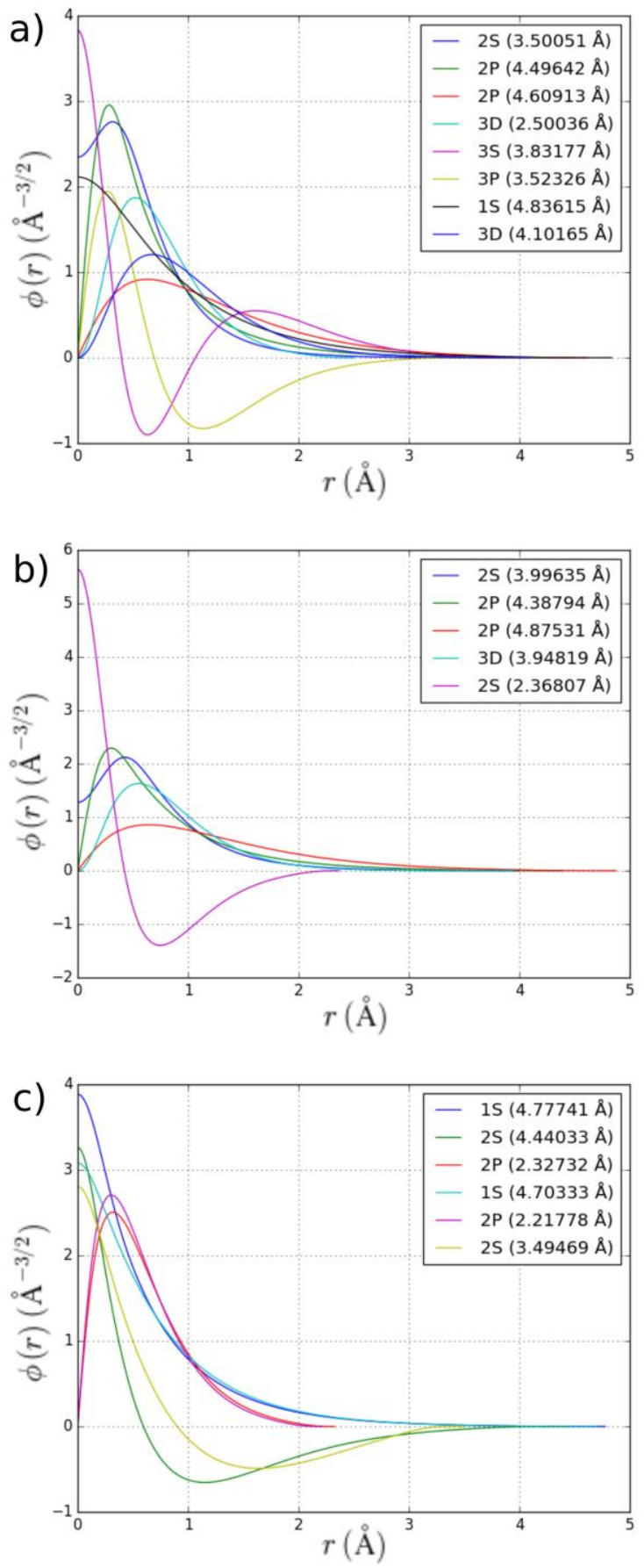

Fig. 3. Basis sets visualization for a) nitrogen, b) hydrogen, c) carbon. 


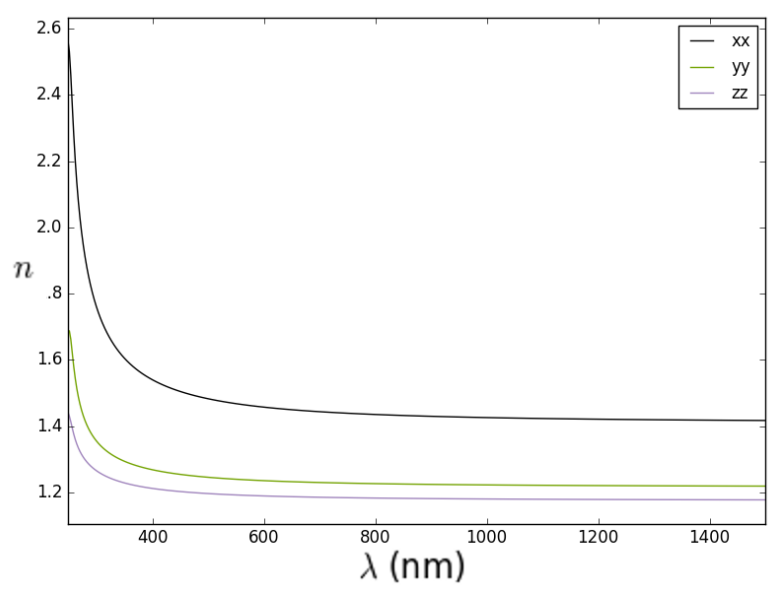

Fig. 4. Refractive index for 3 basic geometrical directions X, Y, Z.

Relying on the DFT calculation of electron structure, optical properties were calculated. In Fig. 4, we see the plot of refraction index vs. wavelength. We can see that the character of dispersion is normal. The change in the reflection index and absorption index is still normal in a wavelength range from $300 \mathrm{~nm}$ over $1000 \mathrm{~nm}$. Refractive index values show good agreement with the experimental values of $n=1.37$ [7]. As we can see in Fig. 4, the refractive index is dependent on molecule direction therefore the result may be different because it should be taken into account that a medium refractive index from different directions should give an efficient approximation.

DFT calculations provide a powerful tool to predict material properties accurately. The results presented in this paper show that we can use the LCAO DFT with a periodic boundary condition approach to organic molecules and obtain a physical result. Moreover, it is possible, due to a careful choice of pseudopotential, to achieve optical parameters such as the refractive index with precision similar to that of the B3LYP method [14]. The importance of this information comes from the fact that the usage of B3LYP is far more resources consuming than LCAO DFT GGA and enables to study much bigger molecules and systems. In the context of the obtained results, it is possible to think about calculating the electrooptical properties for allylamine adsorbed to a semiconductor surface.

The authors gratefully acknowledge the financial support from the Polish National Science Centre (NCN) under Grant No. 2016/21/B/ST7/01430, 2016/22/E/ST7/00102, 2014/14/M/ST5/00715 and National Centre for Science and Development Grant Techmatstrateg No. 347324. This work was partially supported by the Science for Peace
Programme of NATO (Grant no. G5147). The DS funds of the Faculty of Electronics, Telecommunications, and Informatics of the Gdansk University of Technology are also acknowledged.

\section{References}

[1] W. Kohn, L.J. Sham, Phys. Rev. 140(4A), A1133 (1965).

[2] J.P. Perdew, K. Burke, M. Ernzerhof, Phys. Rev. Lett. 77(18), 3865 (1996)

[3] L.G. Ferreira, M. Marques, L.K. Teles, Phys. Rev. B 78(12), 125116 (2008).

[4] L.G. Ferreira, M. Marques, L.K. Teles, AIP Advanc. 1(3), 032119 (2011).

[5] M. Schlipf, F. Gygi, Computer Phys. Comm. 196, 36 (2015).

[6] P. Prayongpan, C. Michael Greenlief, Surf. Scien. 603(7), 1055 (2009).

[7] M.T. van Os, B. Menges, R. Foerch, G.J. Vancso, W. Knoll, Chem. Mat. 11(11), 3252 (1999).

[8] J. Zeng, R.-Q. Zhang, H. Treutlein, Quantum Simulations of Materials and Biological Systems (Springer Science \& Business Media, 2012).

[9] I. Del Villar, I.R. Matias, F.J. Arregui, Opt. Lett. 30(18), 2363 (2005).

[10] D. Nidzworski et al., Scien. Rep. 7, 15707 (2017).

[11] Synopsys QuantumWise, Atomistix Toolkit version 2018.06

[12] D.C. Liu, J. Nocedal, Math. Programm. 45(1-3), 503 (1989).

[13] K.F. Garrity, J.W. Bennett, K.M. Rabe, D. Vanderbilt, Comput. Mater. Science 81, 446 (2014).

[14] Y. Cai, T. Zhang, A.B. Anderson, J.C. Angus, L.N. Kostadinov, T.V. Albu, Diamond Related Mat. 15(11), 1868 (2006). 